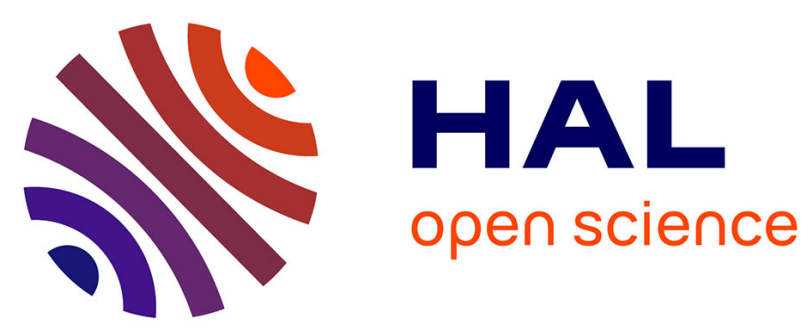

\title{
A MULTIDISCIPLINARY APPROACH TO OVERREACHING DETECTION IN ENDURANCE TRAINED ATHLETES
}

Yann Le Meur, Christophe Hausswirth, Françoise Natta, Frank Bignet, Pierre Vidal

\section{To cite this version:}

Yann Le Meur, Christophe Hausswirth, Françoise Natta, Frank Bignet, Pierre Vidal. A MULTIDISCIPLINARY APPROACH TO OVERREACHING DETECTION IN ENDURANCE TRAINED ATHLETES. Journal of Applied Physiology, 2012, 114 (3), pp.411-420. 10.1152/japplphysiol.01254.2012 . hal-01835107

\section{HAL Id: hal-01835107 https: / hal-insep.archives-ouvertes.fr/hal-01835107}

Submitted on 11 Jul 2018

HAL is a multi-disciplinary open access archive for the deposit and dissemination of scientific research documents, whether they are published or not. The documents may come from teaching and research institutions in France or abroad, or from public or private research centers.
L'archive ouverte pluridisciplinaire HAL, est destinée au dépôt et à la diffusion de documents scientifiques de niveau recherche, publiés ou non, émanant des établissements d'enseignement et de recherche français ou étrangers, des laboratoires publics ou privés. 
4

6

7

8

9

\section{A MULTIDISCIPLINARY APPROACH TO OVERREACHING DETECTION IN ENDURANCE TRAINED ATHLETES}

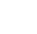

Authors: Yann LE MEUR ${ }^{1 *}$, Christophe HAUSSWIRTH ${ }^{1 *}$, Françoise NATTA $^{1}$, Antoine COUTURIER ${ }^{1}$, Frank BIGNET ${ }^{2} \&$ Pierre Paul VIDAL ${ }^{3}$ 7

*These authors contributed equally to this work.

${ }^{1}$ National Institute of Sport, Expertise and Performance (INSEP), Research Department, Paris, France

${ }^{2}$ French Federation of Triathlon, Saint Denis, France

${ }^{3}$ CESeM, CNRS, University of Paris V, Paris, France

4

Running title: Multifactorial analysis for overreaching detection

\section{Corresponding author:}

Christophe HAUSSWIRTH,

Research Department,

National Institute of the Sport, Expertise and Performance (INSEP),

11 Avenue du Tremblay, 75012 Paris, FRANCE

2 Phone : + 33 1-41-74-43-85

3 Fax : + 33 1-41-74-45-35

4 E-mail : christophe.hausswirth@insep.fr 


\section{AUTHOR CONTRIBUTIONS}

2

3 The experiments were conducted 32 in the laboratory of the National Institute of Sport, Expertise and

4 Performance, Paris, France

5

6 Conception and design of the experiments:

7 Yann Le Meur, Christophe Hausswirth, Françoise Natta, Antoine Couturier, Frank Bignet \& Pierre Paul

8 Vidal

9

10

Collection of the data

11 Yann Le Meur, Christophe Hausswirth, Françoise Natta, Antoine Couturier, Frank Bignet

12

13 Analysis and interpretation of data:

14 Yann Le Meur, Christophe Hausswirth, Françoise Natta, Antoine Couturier, Frank Bignet \& Pierre Paul

15 Vidal

16

17 Drafting the article or revising it for important intellectual content:

18 Yann Le Meur, Christophe Hausswirth, Françoise Natta, Antoine Couturier, Frank Bignet \& Pierre Paul

19 Vidal

20

21 All authors approved the final version of the manuscript. 
3 In sport, high training load required to reach peak performance push human adaptation to their limits.

4 In that process, athletes may experience general fatigue, impaired performance and may be identified

5 as overreached (OR). When this state lasts for several months, an overtraining syndrome is diagnosed

6 (OT). Until now, no variable per se can detect $\mathrm{OR}$, a requirement to prevent the transition from OR to

7 OT. It encouraged us to further investigate OR using a multivariate approach including physiological,

8 biomechanical, cognitive and perceptive monitoring. Twenty-four highly trained triathletes were

9 separated into an overload group and a normo-trained group (NT) during three weeks of training.

10 Given the decrement of their running performance, eleven triathletes were diagnosed as OR after this

11 period. A discriminant analysis showed that the changes of eight parameters measured during a maximal incremental test could explain $98.2 \%$ of the OR state (lactataemia, heart rate, biomechanical parameters and effort perception). Variations in heart rate and lactataemia were the two most

14 discriminating factors. When the multifactorial analysis was restricted to these variables, the 15 classification score reached 89.5\%. Catecholamines and creatine kinase concentrations at rest did not 16 change significantly in both groups. Running pattern was preserved and cognitive performance 17 decrement was observed only at exhaustion in OR subjects. This study showed that monitoring various 18 variables is required to prevent the transition between NT and OR. It emphasized that an OR index, 19 which combines heart rate and blood lactate concentration changes after a strenuous training period, could be helpful to routinely detect OR. 
Increases in training and volume are typically undertaken by athletes in an attempt to enhance

4 physical performance. High training loads (i.e. increased training volume and intensity) can place significant stress on the athlete's cognitive and physiological systems and if not matched by appropriate rest/recovery can lead to maladaptation, leading to increased fatigue and reduced performance $(30,41)$. When athletes require several days or weeks to recover physical performance, they are diagnosed as being overreached (OR) (30). Common symptoms reported with OR include general fatigue, sleep disorders, decreased appetite, loss of body weight, anxiety, reduce motivation, lack of concentration and variation of mood (18). In severe cases of maladaptive training, known as overtraining (OT), athletes may have reduced performance capacity either with or without these clinical symptoms that remain for several months or years. This most severe form of training maladaptation presents a serious threat for athletic performance and health. The currently accepted method for diagnosing OR/OT is to monitor performance after completion of a resting period of several days or weeks (18). Nevertheless, this method is frequently rejected by coaches and athletes because it may endanger the training continuum and it could lead to potential detraining. It is therefore important to identify early markers of OR/OT to limit the occurrence of these training maladaptation forms in population at risk.

Many physiological variables have been recorded to detect OR and OT. One of the most reported physiological measures in endurance athletes has been a right shift in the lactate curve $(4,16$, $22,28,39,44)$. However, it has not been reported by all investigators $(10,26)$. Similarly, decreased nocturnal urinary catecholamine excretion has been associated with OT in endurance athletes and interpreted as lowered intrinsic sympathetic activity $(25,29)$. Nevertheless, a reduced intrinsic sympathetic activity has not been observed in all studies investigating OR/OT $(19,44,46)$. A decrease in the ratio between the hormones testosterone or free testosterone and cortisol has also been proposed as a physiological marker of "anabolic-catabolic balance", a putative tool in the diagnosis of OT (1). Again, not all studies have observed changes in these variables with OR/OT (25, 29, 43, 46), and therefore, they are not considered as a good independent measure of maladaptive training (18). Finally, changes in heart rate (HR) at rest, and during both submaximal and maximal exercise have been reported to be associated with $\mathrm{OR}$ in various sports $(9,10,19,22,26,39)$. However, a recent meta-analysis examining the effect of overload training on resting, submaximal and maximal exercise HR and heart rate variability demonstrated that the small to moderate changes in these variables limits their clinical usefulness as idiosyncratic markers of OR and OT (5). Altogether then, the lack of consensus amongst research suggests that independent physiological markers may have limited practical usefulness if used as early warning markers of OR/OT. 
In that context, there has been increasing interest in the application of cognitive tests as early warning measures of both OR and OT athletes (12, 13, 21, 31, 32). Nederhof et al. (32), reported that executive functions can be influenced by training tolerance and suggested that alterations in these functions may be an early indicator of maladaptive physical training. This hypothesis was strengthened by three studies that reported small increases in response time and increased number of mistakes in Stroop test at rest in OR and OT athletes $(12,13,21)$. It remained that large interindividual variability in the results of the cognitive tests limited their usefulness to assess a state of OR, especially when used alone. Also, cognitive performances had been assessed at rest and not during exercise, which could be a more suited measure to detect maladaptation in athletes.

In summary, investigations into early warning markers of OR / OT was still elusive and 49 idiosyncratic physiological, biomechanical and cognitive variables that could identify OR remained to be found $(18,35,45)$. It led us to propose a multivariate approach to identify athletes at risk of OR/OT. In order to test that hypothesis, we simultaneously monitored physiological, cognitive and biomechanical parameters at rest and during exercise in athletes progressively driven to OR by a prolonged period of overload training. We chose triathletes because they often undertake heavy loads during training and therefore have been reported to be at risk of OR and OT.

\section{Methods}

\section{Ethical approval}

Twenty-four well-trained triathletes volunteered to participate in this study. All subjects had competed in triathlons for at least 2 years and were training a minimum of 6 times per week. The experimental design of the study was approved by the Ethical Committee of Saint-Germain-en-Laye (acceptance no. 10054) and was done in accordance with the Declaration of Helsinki. Prior to participation in the investigation, subjects underwent medical assessment. After comprehensive verbal and written explanations of the study, all subjects gave their written informed consent.

The subjects were randomly assigned to either the experimental group (intensified training (IT) group) or the control group (normal training group, NT) according to a matched group experimental design based on maximal oxygen uptake ( $\left.\mathrm{V}_{2 \max }\right)$ and maximal aerobic speed (MAS). Subjects' characteristics are presented in Table 1.

\section{Experimental protocol}


The protocol is illustrated in Figure 1. The investigation was conducted in September/October at the end of the competitive triathlon season to ensure a high fitness level for all participants. The training of each triathlete was monitored for a period of 7 weeks in total, which was divided into three distinct phases. The two first phases were similar for both IT and NT groups. The first phase (I) consisted of 3 weeks during which the subjects completed their usual amount and type of training (classic training). The second phase (II) consisted of one week of moderate training during which the subjects were asked to divide their normal training week by a half (recovery week). During the third period (III), the IT group completed a 3-week intensified program designed tp deliberately overreach the triathletes; the duration of each training sessions of the classic training period was increased by $40 \%$. The NT group reproduced its classic training program during the same period. Throughout the entire experiment, the same sport scientist coached all triathletes. Training schedule was controlled to remain similar during each week of phase III. To avoid injuries, particular attention was devoted to daily feedback obtained from the triathletes. Throughout the entire study, heart rate was recorded during training to ensure that the triathletes adhered to prescribed training. At the end of phases II and III, the triathletes performed a maximal incremental running test on a 340-m indoor running track. To ensure that performance variations during the maximal incremental runs were due to the global training regimen and not to the training session(s) performed the day before each test, the subjects were required to respect a $24 \mathrm{~h}$ rest period before each maximal incremental run session.

\section{Assessment of energy intake}

During the $48 \mathrm{~h}$ prior, each maximal oxygen uptake ( $\mathrm{VO}_{2 \max }$ ) test, the triathletes were required to follow a nutritional plan in order to ensure muscle glycogen store resynthesis. They were allowed access to a buffet-type array of breakfast and meals foods and instructed to eat until satiety was reached. Breakfast consisted of a variety of macronutrients from both solid and liquid energy sources. The selected foods included an assortment of cereals, bread, fruit, yogurt, milk, juice, ham and cheese. In the lunch and dinner meals, athletes ate a mixed salad as starter, then white meat during lunch and fish during dinner. The side plate consisted of a mixed of 50\% carbohydrates (i.e., pasta, nice, noodles) and 50\% of vegetables (i.e., green beans, broccoli, tomatoes). One fruit and one yogurt were added as dessert, for lunch and dinner.

\section{Maximal running test}

The triathletes completed a maximal incremental running test on a 340-m indoor track to

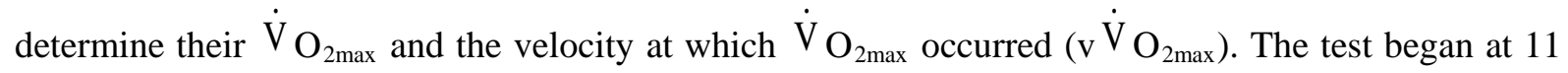


$110 \mathrm{~km} \cdot \mathrm{h}^{-1}$ and the speed was increased by $1 \mathrm{~km} \cdot \mathrm{h}^{-1}$ every 3 minutes until volitional exhaustion. A rest

111 period of 1-min was provided between each running step. The triathletes followed a cyclist travelling

112 at the required velocity to ensure that the subjects were respecting the imposed pace. Visual marks

113 were set at $20 \mathrm{~m}$ intervals along the track. The cyclist received audio cues via an mp3 player; the cue

114 rhythm determined the speed needed to cover $20 \mathrm{~m}$. The coefficient of variation of running speed

115 between the tests pre- and post-phase III for each running step was subsequently calculated in order to

116 assess the reproducibility of this parameter between the two tests.

117

118

119

120

121

122

123

124

125

126

127

128

129

130

131

132

133

134

135

136

137

138

139

140

141

142

143

\section{Physiological parameters}

Peripheral venous blood samples were taken from an antecubital vein of participants before each running test. Samples were drawn into non-additive tubes under sterile conditions. Serum was separated from whole blood by centrifugation at $1,000 \mathrm{~g}$ for $10 \mathrm{~min}$ at room temperature. An OLYMPUS 2700 analyzer (Beckman Coulter, Brea, USA) was used for simultaneous assay with reagents from the manufacturer of Creatine Kinase (CK). Plasma adrenalin and noradrenalin were measured in high-performance liquid chromatography with electrical detection (Laboratoire Medibio, Montargis, France).

\section{Metabolic parameters}

Between each increment, blood samples were taken from the participants' ear lobes during a 1-min rest period and analyzed using a Lactate Pro system (36). Oxygen uptake ( $\dot{\mathrm{V}}_{\mathrm{O}_{2}}$ ) and expiratory flow $\left(\dot{\mathrm{V}}_{\mathrm{E}}\right.$ ) were recorded breath-by-breath with a telemetric system collecting gas exchanges (Cosmed K4b², Rome, Italy) (11), which was calibrated before each test. Heart rate values (HR) were monitored every second using a Polar unit. Expired gases and HR values were subsequently averaged every $5 \mathrm{~s}$ and were analysed (i.e., mean value) on time periods corresponding to the last 30s of each running step. $\dot{\mathrm{V}}_{\mathrm{O}_{2 \max }}$ was determined at exercise cessation when a plateau in $\dot{\mathrm{V}}_{\mathrm{O}_{2}}$ despite an increase in running speed was observed. If the subjects did not demonstrate any plateau in $\dot{\mathrm{V}}_{\mathrm{O}_{2}}$, the test was considered to be maximal, when the respiratory exchange ratio value exceeded 1.15 and maximal HR value was over $90 \%$ of the predicted maximal value. The lactate threshold (LT) was assessed according to the D-max method previously described by Cheng et al. (7).

\section{Biomechanical parameters}


Kinetic measures. An area of biomechanical data collection was installed in a particular 145 location of the indoor running track. This area was equipped with six adjacent force platforms 146 (Z2074AA, Kistler, Switzerland) embedded in the track and covered with a layer of tartan, so as to not 147 influence or disturb the triathletes while running. The total platform surface was approximately $6.6 \mathrm{~m}$ 148 long and $0.6 \mathrm{~m}$ wide and the output signals of the six platforms were acquired in series at $1000 \mathrm{~Hz}$. 149 This length enabled data recording of at least four leg support phases (two left-side and two right-side supports) regardless of the running speed. This device gathered, for each instant of the support phase, 151 the lateral $(\mathrm{Fx})$, anteroposterior $(\mathrm{Fy})$ and vertical $(\mathrm{Fz})$ components of the force exerted by the 152 triathletes on the ground. The data collected were propulsion (PImn) and braking impulses (BImn), 153 peak vertical impact (Rz1n), maximum peak vertical force (Rz2n), support (dS), aerial (dA) and 154 braking durations ( $\mathrm{dBn}$ ). Impulses and forces were normalized to body weight (x 1000 for impulses). 155 Braking duration was normalised to support duration.

Kinematic measures. The movement acquisition system was a Vicon optoelectronic device (Oxford, United Kingdom), which uses 12 T10 cameras (resolution: 1megapixels) to follow and record in 3D the position of set retroreflective (passive) spherical markers. The acquisition frequency was set at $200 \mathrm{~Hz}$. To reduce the effects of sliding of the markers, the triathletes were dressed in tight fitting outfits and markers were fixed with double-sided tape and their contact was reinforced with elastic adhesive strips.

Recordings from the force-platform and the video acquisition systems were synchronized. Depending on the running speed, the triathletes ran between one and three times in this area. The data collected were step length (Lxn) and width (Lyn), which were normalized to leg length and analyzed using mean values for each running stage.

\section{Cognitive performance.}

During the maximal incremental running test, subjects had to respond to audio stimuli occurring in the second half of each 3-minute running stage.

Double-task. The system was comprised of two modified nunchuks (Nintendo WII, Tokyo, Japan), an mp3 player and recorder, earphones and linking audio cables. Nunchuks were chosen based upon their light-weight and ergonomic design. To avoid any confusion, the upper analog stick was removed, the middle finger button was locked in the pressed down position and only the forefinger

177 button was kept functional. Custom electronics allowed forefinger button actions to be recorded along with the given audio stimuli. The whole system weighed approximately $70 \mathrm{~g}$.

Audio stimuli were delivered through earphones and consisted of 30 single and double, high- 
181

182 double high-pitched tone, the triathlete was required to press down the left nunchuk button. Upon 183 hearing a single high-pitched or double low-pitched tone, the triathlete was required press the right 184 nunchuk button. All triathletes were instructed to respond as fast as possible. One week before the first maximal incremental running test, they received an mp3 test file for training, and repeated this training prior to each maximal incremental running test.

High- and low-pitched tones were respectively set as $5000 \mathrm{~Hz}$ and $150 \mathrm{~Hz}$ sine waves. Such frequencies allowed the triathletes to unequivocally distinguish high- from low-pitched tones. Single tones consisted of a $200 \mathrm{~ms}$ sine wave and double-tones consisted of two $70 \mathrm{~ms}$ sine waves interspaced with $80 \mathrm{~ms}$, which resulted in a $220 \mathrm{~ms}$ stimulus. Such durations made it impossible for 190 the triathletes to initiate any decision process before they had heard the entire stimulus.

191

It is well established that perceived loudness depends on tone $(15,37)$ and duration $(33,34)$.

192 Single and double, high- and low-pitched tones amplitudes were adjusted in accordance to equal193 loudness contours (often referred to as Fletcher-Munson curves) so that they met the international 194 standard ISO 226 specifications (ISO 2012). During the medical assessment, subjects underwent an 195 audiogram to ensure none of them had any hearing impairment.

The 30 stimuli were introduced in random order into a $90 \mathrm{~s} \mathrm{mp3} \mathrm{file} \mathrm{and} \mathrm{were} \mathrm{separated} \mathrm{with} \mathrm{a}$ random duration such that two consecutive stimuli were interspaced by at least $500 \mathrm{~ms}$. A different file was played for each running stage so that it was not possible for the subject to learn the stimuli arrangement inside a file.

Data were processed in OriginPro 8.1 (OriginLab, Northampton, MA) with a custom-written script that returned, for each running stage, the percentage of false answers (excluded $<200 \mathrm{~ms}$ ).

Questionnaires. The effect of the training regimen was also recorded through the assessment of the perceived sensations of subjects. The subjects were tested at rest and during the maximal incremental tests.

The Mindeval system was used to collect the data at rest (Mindeval GydleInc. Québec, CANADA). It is comprised of a web interface with a database and a stand-alone application. In the Pre- and -Post conditions, participants entered their personal key and answered questions within three areas related to pain, tiredness, and well-being, using a visual analogic scale. The software records the location of the indicator with a number ranging between 0 (no pain) and 100 (maximum pain). The collected data was stored on a secured server. Before the initiation of the study, triathletes were accustomed to the software, and the questions relative to their subjective sensations were thoroughly explained.

The rating of perceived exertion (RPE) was measured verbally using the Borg scale (3) during the maximal running test. This scale measures the subjective sensations accompanying the exercise. The scale and its purpose were carefully explained to each triathlete before each incremental test. The 
triathletes were instructed to give a general RPE, a muscular RPE and a ventilatory RPE, immediately at the end of each running step and at exercise cessation.

\section{Data and statistical analyses}

The effect of the training regimen was analysed using the magnitude of variation between the beginning and the end of phase III for every parameter investigated. To reduce the effect of interindividual differences in performance level, subsequent analyses were performed for three relative intensity levels of exercise determined for each triathlete at the end of phase III: low intensity running, lactate threshold (LT) and at exhaustion. Each parameter was compared with its respective value measured for the same running speed at the beginning of phase III. For all triathletes, the low intensity running was set at $13 \mathrm{~km} \cdot \mathrm{h}^{-1}$ because: i) A very low coefficient of variation of running speed was indeed reported until this intensity (coefficient of variation of 3.93 and 2.24 at $12 \mathrm{~km} \cdot \mathrm{h}^{-1}$ and $13 \mathrm{~km} \cdot \mathrm{h}^{-1}$, respectively); ii) this running velocity was at least $2 \mathrm{~km} \cdot \mathrm{h}^{-1}$ lower than LT for all triathletes.

Statistical analysis was performed using Statistica software for Windows (Statsoft, version 7.0, Statistica, Tulsa, Oklahoma, USA). For the statistical procedure, the level of significance was set at $\mathrm{p}<0.05$.

Assessment of the OR syndrome. In order to determine the reproducibility of performance during the maximal running test and to identify OR athletes in the IT group, ICC (intraclass correlation coefficient) and confidence interval at $100 \%$ of performance variation were calculated for the NT group. To be diagnosed as OR, athletes of the IT group had to reveal a performance decrement higher than the lowest reproducibility value reported for the NT group (OR threshold). Using that procedure, the IT group was divided in two subgroups. When the subjects of the IT group demonstrated a performance decrement higher than OR threshold, they were considered truly overreached (OR group). When this assumption was not confirmed at the end of the overload period, they we were not considered overreached (n-OR group).

Discriminant analyses

Three stepwise discriminant analyses (DA) were conducted to determine the ability of the 251 different variables measured during exercise to distinguish between NT, n-OR and OR groups and 252 subsequently predict group membership. The criterion used to determine whether a variable entered the model (i.e., discriminant function) was Wilk's Lambda, which measures the deviations within each 
group with respect to the total deviations. The sample-splitting method initially included the variable that most minimized the value of Wilk's Lambda, provided the value of $\mathrm{F}$ was greater than a certain critical value. The next step was pairwise combination of the variables with one of them being the variable included in the first step. Successive steps were performed in the same manner, always with the condition that the F-value corresponding to the Wilk's Lambda of the variable to select has to be greater than the aforementioned "entry" threshold. If this condition was not satisfied, the process was halted, and no further variables were selected in the process. Before including a new variable, an attempt was made to make some of those already selected if the increase in the value of Wilk's Lambda was minimal, and the corresponding F-value was below a critical value. Wilk's Lambda, canonical correlation index, and percentage of subjects were computed as indicators of OR predictive capacity.

The first DA (DA1) was performed on all the tested subjects (NT, n-OR and OR groups: 24 subjects tested at 3 running intensities) using all the variables tested in the study ( $n=21)$. It was used to determine if some variables would allow to identify three groups of triathletes according to their training regimen and their performance decrement during the protocol. The second DA (DA2) excluded the n-OR group (NT and OR groups: 19 subjects at 3 running intensities, see below for the justification of the 19 subjects) using all the variables measured $(n=21)$. This analysis was performed to identify the most valuable variables in classifying triathletes of NT and OR groups as overreached or not. The discriminating variables with their respective Wilk's lambdas and p-value, canonical correlation $\left(\mathrm{r}_{\mathrm{c}}\right)$ and classification percentage were noted. Considering that markers of OR should be applicable in training practice (32), a third additional DA (DA3) was performed to investigate the minimal number of variables allowing a reasonable discrimination between the OR and NT groups.

Parameters evolution

Since this protocol involved a relatively small number of subjects $(n<32)$ and the data obtained did not always meet the assumptions of normality, as assessed visually by normal probability plot and by the Shapiro-Wilk test, non-parametric statistical analyses ensued. A Friedman rank test was undertaken to evaluate the statistical differences in time for each group and a Mann-Whitney test was completed to assess significant differences between NT and OR groups. The results are expressed as the mean value with standard deviation $( \pm S D)$. 

groups.

\section{Assessment of the OR syndrome}

An intra-class correlation test (ICC) was used to classify the subjects from the IT group as overreached (OR group) or non-overreached (n-OR group). First, the reproducibility of the performance of the NT group was measured using the ICC test (see method). ICC value was very high (ICC $=0.98$ ), with a performance repeatability ranging between 0.6 to $1.8 \%$ (mean: $0.9 \%$ ). On the basis of this analysis, a decrement of performance of greater than $1.8 \%$ was used as the criteria to discriminate the OR subjects in the IT group. Subsequent analysis showed that only 11 of the 16 triathletes that complete the overload training were considered as truly OR group). The five other subjects of the IT group were not diagnosed OR.

\section{Performance}

In the OR group, the running performance decreased on average by $4.4 \pm 1.1 \%$ between the beginning and the end of the intensified training period $\left(18.3 \pm 0.2 \mathrm{~km} \cdot \mathrm{h}^{-1}\right.$ and $17.6 \pm 0.3 \mathrm{~km} \cdot \mathrm{h}^{-1}, p<$ 0.001, pre- and post-overload period, respectively). When expressed in total running distance covered during the incremental test, this decline represented $13.3 \pm 3.2 \%$.

\section{Physiological parameters}

Both the NT and OR groups were first submitted to the same initial 4 week training protocol (phases I and II in Figure 1). As shown in Table 2, the physiological variables values measured at the end of phase II were not significantly different between the two experimental groups. The OR group then completed a training program with $40 \%$ increase in load (phase III). lb values was observed for the OR group for the two submaximal intensities and at exhaustion (Table 2a). In contrast, no significant variation was observed for these two parameters for the three running intensities in the NT group. These variations in HR and $\left[\mathrm{La}^{-}\right]_{b}$ values were significantly different for OR and NT groups for all the running intensities (compare the numerical values in columns 3 and 6 of

323 Table 2a). No significant differences in $\dot{\mathrm{V}}_{\mathrm{O}_{2}}$ and $\dot{\mathrm{V}}_{\mathrm{E}}$ values were observed between the two groups 324 before and after phase III. 
Blood parameters. No significant statistical difference in [CK] was observed in the OR group during phase III (234 \pm 142 and $257 \pm 157$ UI.L ${ }^{-1}$, pre- and post- phase III, $p=0.07$ ). No significant variation was observed either in the NT group for this parameter during the same period (180 \pm 83 and $161 \pm 49$ UI..$^{-1}$, pre- and post- phase III, respectively, $p=0.48$ ). Similarly, there were no significant differences in plasma catecholamine concentrations in both groups before and after phase III ( $p>$ 0.37). Similarly, there were no significant interaction (time $x$ training regimen) for plasma $[\mathrm{CK}](p=$ $0.17)$, adrenalin $(p=0.88)$ and noradrenalin $(p=0.90)$ at rest.

\section{Cognitive performance}

There was no difference between groups at rest (-5.5 $\pm 11.2 \%,-4.3 \pm 3.4 \%$, for NT and OR groups, respectively, $p=0.39)$, low intensity $(-1.2 \pm 4.5 \%,-2.0 \pm 5.5 \%$, for NT and OR groups, respectively, $p=0.69)$ and lactate threshold $(-1.9 \pm 8.7 \%, 1.3 \pm 9.2 \%$, for NT and OR groups, respectively, $p=0.52$ ). In contrast, the OR group demonstrated a significant decrease in performance at exhaustion than the NT group ( $8.7 \pm 11.3 \%$ and $-12.1 \pm 17.9 \%$, for NT and OR groups, respectively, $p=0.04)$.

\section{Biomechanical parameters}

Except dS (support duration) at LT (lactate threshold) (-11 $\pm 12 \mathrm{~ms}$ and $2 \pm 6 \mathrm{~ms}$, for OR and NT groups, respectively, $p=0.01$ ), no significant interaction effect was reported for all the 9 parameters investigated at three running speeds $(p>0.05)$ (Table $2 b)$.

\section{Perceived sensations}

At rest

The OR triathletes reported increased sensations of pain (16 \pm 24 and $53 \pm 26, p<0.01$, before and after the overload period, respectively) and tiredness (20 \pm 18 and $85 \pm 11, p<0.001$, before and after the overload period, respectively). In contrast, there was no significant difference for these two parameters during the same period for the NT group (28 \pm 32 and $18 \pm 13$, for pain, $38 \pm 16$ and $38 \pm$ 24 , for tiredness, before and after phase III, respectively, $p>0.05$ ). There was a significant difference in the change in pain $(p=0.03)$ and tiredness $(p<0.001)$ between the OR and NT groups. Well being sensation demonstrated no significant change in both groups before and after phase III (76 \pm 17 and 61

$360 \pm 31, p=0.23$, for OR group, $73 \pm 22$ and $73 \pm 20, p=0.72$, before and after the overload period, respectively). 
There was a significant difference in $\triangle$ GenRPE (general perceived exertion change) was observed at exhaustion ( $+1.8 \pm 1.4$ and $+0.1 \pm 1.3, p=0.02$ ) between the OR and NT groups, however there were no-statistical differences at low $(+2.1 \pm 3.1$ and $-0.4 \pm 1.0, p=0.05)$ and LT intensities $(+2.2 \pm 2.4$ and $+0.1 \pm 1.8, p=0.08$ ). The $\triangle$ MuscRPE (muscular perceived exertion change) was significantly different between NT and OR groups at Low $(+4.1 \pm 3.2$ and $+0.0 \pm 1.0, p<0.01)$ and LT intensities ( $+3.3 \pm 2.2$ and $+0.8 \pm 1.1, p=0.02)$, but not at exhaustion $(+3.3 \pm 2.0$ and $+1.7 \pm 1.4, p$ $=0.10$ ). Finally, the training load did not influence $\triangle$ VentRPE (ventilatory perceived exertion change) for the three running intensities $(p>0.20)$.

\section{Discriminant analyses}

The DA1 was performed on all the tested subjects using all the variables tested in the study. It was used in order to determine if some variables would allow identification of three groups of triathletes according to their training regimen and performance decrement during the protocol. DA1 indicated the presence of two significant discriminant functions $(\mathrm{p}<0.01)$. As a linear combination of discriminating variables, the analysis resulted in canonical coefficients for the first function being derived so that the group means on the function were as different as possible. The coefficient for the second function was also derived to maximize the differences between the group means as long as the values on the second function were not correlated with those on the first function. The discriminant functions were used to compute the position of the triathlete's data in the discriminant space (Figure 2). The horizontal direction corresponded to function 1, with the lateral separation among the three groups indicating how much they were distinguished on this function. The vertical axis corresponded to function 2, with the vertical separation indicating the manner in which the groups were distinguished in a way unrelated to the way they were separated on function 1 (40). Using this analysis 87.5\% of the NT, n-OR and OR subjects were classified in the correct group (Table 3). With three groups, 33.3\% of correct predictions are possible with pure random assignment (24). In summary, DA1 showed that we could discriminate the three groups of athletes using the variables measured.

The second DA (DA2) excluded the n-OR group using all the variables measured. It was performed to identify the most valuable variables in classifying triathletes of NT and OR groups as overreached or not. It indicated the presence of one significant discriminant function $(p<0.001)$. The discriminant function was interpreted by examining the standardized coefficients (see Table 4a) in order to ascertain which variables contributed most to determining scores on the function. The larger the magnitude of the coefficient, the greater the contribution of that variable to the discriminant function. $\triangle \mathrm{HR}$ (heart rate variation) made the greatest contribution to scores on that function followed 
by $\Delta \mathrm{dS}$ (stance phase duration change), $\Delta \mathrm{dA}$ (aerial phase duration change), $\Delta\left[\mathrm{La}^{-}\right]_{\mathrm{b}}$ (blood lactate concentration change) and $\Delta \mathrm{Lxn}$ (step-length change) with a lesser contribution from the three other factors selected in the model $(\Delta \mathrm{PImn}$, propulsive impulse change; $\Delta \mathrm{Lyn}$, step largeness change; $\Delta$ muscRPE, muscular perceived exertion change). The classification procedure correctly placed $98.2 \%$

404

405

406

407

408

409

410

411

412

413

414

415

416

417

418

419

420

421

422

423

424

425

426

427

428

429

430

431

432

433

434

435

436

of the triathletes of NT and OR groups into their respective groups (see Table $4 \mathrm{~b}$ ). The probability by chance with two groups would have been $50.0 \%$. The extent to which all parameters were valuable and necessary in DA2 was determined via a stepwise procedure. A forward stepwise procedure was utilized whereby the individual variable that provided the greatest univariate discrimination was selected first and was then paired with each of the remaining variables one at a time, to determine the combination which produced the greatest discrimination. This analysis included the 8 selected variables of DA2 in the following order of decreasing discriminating power: $\Delta \mathrm{HR}, \Delta\left[\mathrm{La}^{-}\right]_{\mathrm{b}}, \Delta \mathrm{PImn}$, $\Delta \mathrm{dS}, \Delta \mathrm{dA}$ and $\Delta \mathrm{Lxn}$. All these variables made a significant $(p<0.05)$ contribution to discrimination between NT and OR groups, while no statistical significant contribution were observed for both $\Delta$ Lxn and $\triangle$ MuscRPE (Table $4 \mathrm{c}$ ). In summary, DA2 ranked 8 of the 21 variables measured as valuable to discriminate between OR and NT groups.

Considering that only a limited number of markers of OR could practically be applied in the training environment, a third additional DA (DA3) was performed. It investigated the minimal number of variables allowing a reasonable discrimination between the OR and NT groups. When the variables was restricted to $\Delta \mathrm{HR}$ and $\Delta\left[\mathrm{La}^{-}\right]_{\mathrm{b}}$ (i.e., the two most valuable variables in DA2), the classification score still reached $89.5 \%$ (Table 5). The classification function coefficients determined by DA3 could be used in an equation to determine the likelihood of an individual triathlete to be classified as OR using variables measured during exercise:

OR index $=0.17 \times \Delta \mathrm{HR}+0.89 \times \Delta\left[\mathrm{La}^{-}\right]_{\mathrm{b}}+1.36$

Where $\Delta \mathrm{HR}$ and $\Delta\left[\mathrm{La}^{-}\right]_{\mathrm{b}}$ represent heart rate and blood lactate concentration changes, respectively. As illustrated in Figure 2, using that formalism, a negative value strongly suggests a state of OR.

\section{DISCUSSION}

The main findings of this study were that: (i) Combining physiological, biomechanical and cognitive variables were useful to assess overreaching (OR) in endurance trained athletes after an overload period; (ii) multidimensional analysis showed that heart rate and blood lactate concentration changes were the most important factors in discriminating between control and OR athletes; (iii) while motor control did not appear to be altered during an incremental running test with OR, cognitive 
437 performance was impaired at exhaustion in OR subjects compared to the controls; (iv) the 438 physiological perturbations associated with OR were coherent with perturbations of the autonomic nervous system activity; (v) these results led to the proposal that an index based on two variables could assist in the diagnosis of OR in endurance athletes.

441

442

443

444

445

446

447

448

449

450

451

452

453

454

455

456

457

458

459

460

461

462

463

464

465

466

467

468

469

470

471

472

473

At the end of the overload training period, a $4.4 \%$ decline in maximal running speed was observed in the OR group. Given that the daily variation of this test was $<1.8 \%$ in the NT group, the decline in performance could be attributed to the effects of the intensified training protocol. This reduction in performance was in line with the $5.4 \%$ decrement reported by Halson et al. (17) in OR cyclists with a similar incremental protocol. When expressed in total running distance during the incremental test, this decrease in performance represented 13.3\% in the OR group. A similar decrease was observed by Lehmann et al. (26), who showed an $8 \%$ decline in total running distance during an incremental exercise test in middle- and long-distance runners. Additionally, in our study, the OR triathletes reported a large increase in perceived fatigue at rest, while no significant variations were assessed in the NT group. Reduced physical performance and increased fatigue are two of the common criteria for diagnosing OR (18), which confirmed that these athletes were not adapting to the prescribed overload training. It allowed us to conduct further comparison with the NT athletes (i.e., normal training group) to determine discriminate markers of OR/OT.

\section{Early detection of overreaching}

The aim of this study was to identify specific marker(s) of OR in triathletes that could be used prospectively to prevent endurance athletes from developing OT. The present results showed that a combination of 8 physiological, cognitive and biomechanical parameters changes measured during an incremental maximal running test successfully discriminated between OR and NT triathletes at 98.2\% (chance probability: 50\%). Indeed, with the exception of only 1/57 cases (19 triathletes, 3 running intensities), the training state of individual athletes was adequately classified. Interestingly, the stepwise discriminant analysis indicated that the $\Delta \mathrm{HR}$ and $\Delta\left[\mathrm{La}^{-}\right]_{\mathrm{b}}$ were the two most valuable factors to discriminate between OR and NT groups. When the discriminant analysis was restricted to these two parameters, $89.5 \%$ of the triathletes were still well classified. These findings have strong practical applications as both these measures fulfil the criteria defining a usable marker for detecting OR (and OT) (32): (i) objective; (ii) not easily manipulated; (iii) applicable in training practice; (iv) not too demanding for athletes; (v) affordable for the majority of athletes and (vi) based on a theoretical framework.

We expected that alterations of the running motor patterns (i.e. stride kinematic and mechanical parameters) in triathletes could have been a valid indicator of OR. Surprisingly, we were 
only able to detect minor modifications in the motor pattern, which used in isolation, did not distinguish OR athletes from the NT group. These observations suggest that motor control was largely preserved during the incremental exercise (at submaximal levels), regardless of training status. These findings may also partly explain why athletes can become OR/OT despite close and regular observation from coaches. Indeed, without clearly visible changes in motor patterns (i.e. noticeable changes gait), it becomes difficult to discriminate OR from other potential causes of performance decrement, which emphasizes the necessity for regular monitoring in endurance athletes, especially during periods of heavy training (43). On the basis of the present findings, we suggest to monitor HR and blood lactate concentration. Indeed, the combination of these two measures in the OR index algorithm (OR index $\left.=0.17 \Delta \mathrm{HR}+0.88 \Delta\left[\mathrm{La}^{-}\right]_{\mathrm{b}}+1.36\right)$, could be used as an objective early warning for maladaptive training in endurance athletes.

\section{Underlying mechanisms of overreaching}

The autonomic hypothesis

Whilst the underlying cause(s) of OR (and OT) in endurance athlete remains to be determined $(18,45)$, there is an agreement that the concomitant decrease of $\mathrm{HR}$ and $\left[\mathrm{La}^{-}\right]_{\mathrm{b}}$ reported in several studies could reveal a down-regulation of the sympathetic nervous system and/or changes in parasympathetic/sympathetic tone during OR $(19,26,43)$. Two mutually non-exclusive mechanisms (i.e. centrally and peripherally mediated factors) have been suggested to underpin these physiological changes. In favor of a centrally mediated factors, Lehmann et al. (26) reported decreased nocturnal urinary norepinephrine and epinephrine excretion after an increase in training volume leading to OR. There was also a concomitant decline in submaximal and maximal heart rates along with the changes in catecholamines. In contrast, others reported decreases in heart rate and/or (20) lactate concentration in absence of catecholamine modulations $(17,43)$. Prolonged exposure to catecholamines resulting from intensified training and/or psychological stress may also downregulate $\beta$-adrenergic receptors sensitivity, and/or decrease their number $(27,47)$. This has been observed after exhaustive dynamic exercise (6), chronic exposure to hypoxia (14) and during a prolonged long-term period of heavy endurance training (23) or after infusion of adrenergic agonists (42).

A role for cognitive factors?

In the present study, the cognitive performance was preserved in all athletes at rest and submaximal intensities. Notably however, cognitive performance was reduced at exhaustion in OR athletes. These findings show that whilst cognitive measures were only marginally useful to predict

508 OR, they were affected by OR. These observations are consistent with the threshold theory that 509 involves two hypothetical notions (38). The first suggests that the brain has a reserve capacity and second that the brain has a threshold of impairment. According to this model, the larger the brain 
reserve capacity and the higher the threshold of impairment, the better the tolerance of cognitive

512 processes to different stimuli. In the context of that theory, we propose that the psychological load

513 associated with running during the incremental test (i.e., rate of perceived exertion, RPE) only affected

514 cognitive performance when high running speed were reached (i.e., beyond the lactate threshold). The

515 decreased cognitive performance observed at exhaustion was in agreement with Chmura and Nazar

516 (8), who demonstrated that it is only above lactate threshold that reaction time increased markedly

517 during a running incremental test.

518 The coincidence of increased physical exhaustion and the large deterioration in the double task 519 performance indicated that in OR and NT groups: (i) Running at severe intensities (i.e., above lactate 520 threshold), are accompanied by a large cognitive load; and (ii) that these two tasks rely upon the

521 similar cognitive resources. Moreover, since the cognitive performance showed greater decrease in the

522 OR triathletes (despite lower running speed at exhaustion) than the control group and this occurred

523 with an increase of both general and muscular perceived exertion, it seems that central factors may be

524 involved in OR. This is further supported by the finding that the increased perception of exertion was

525 not associated with higher muscle damage in the OR triathletes. Taken collectively, these results

526 demonstrate that the attention demand of running is increased at high intensity in OR subjects, which

527 may suggest a contribution of central fatigue in OR. These results agree with previous studies that

528 have highlighted similarities between OR/OT athletes with chronic fatigue syndrome and major

529 depression symptoms (2, 32). Indeed, decreased psychomotor speed has consistently been shown to be

530 present in both depression and OR/OT athletes (32). Furthermore, a reduced performance on

531 psychomotor speed tasks was observed in OT athletes at rest $(1212,13,21,31)$. The present

532 investigation extends these results by showing cognitive impairment during strenuous exercise in OR 533 athletes.

\section{Summary}

536 In order to determine discriminant markers of maladaptive training endurance athletes, 537 comparisons were made between various physiological, cognitive and biomechanical measures in OR 538 and non-OR triathletes during 3 weeks of increased training load. A combination of physiological, 539 cognitive and biomechanical parameters changes measured during an incremental maximal running 540 test successfully discriminated between OR and control at 98.2\%. Heart rate and blood lactate 541 concentration variations were the two most discriminating factors (89.5\% of discrimination success, 542 when combined).

543 The results showed that the triathletes running motor patterns were not altered until exhaustion 544 in OR subjects. These observations could explain why athletes can become OR/OT whilst under the 545 close supervision of a coach/scientist. Without visual marker, an external observer would have 546 difficulty to discriminate OR from other potential causes of performance decrement. These findings 547 also highlight that monitoring physiological responses could help preventing OR and OT. On the basis 
548 of the current observations, we propose an OR index, which combines heart rate and blood lactate

549 concentration changes after a training period could be helpful to routinely detect OR in athletes

550 submitted to strenuous training regimen. Indeed, this algorithm may be used to monitor and

551 prospectively guide future manipulations in training load so that the risks of OR/OT are reduced.

552 Whilst the physiological mechanisms that underlie OR/OT remain to be fully elucidated, the 553 concomitant decrease of heart rate and blood lactate concentration changes pointed to perturbations of 554 the autonomic nervous system as one mechanism underlying the genesis of OR. Additionally, since 555 the double task showed that running at severe intensities was accompanied by an increased cognitive 556 load, which is further increased with OR, it also appears that an athlete's cognitive resources are 557 depleted during intense exercise with OR/OT. These results should be now confirmed on a larger 558 population of athletes, involved in different sports and levels of performance. 
1 Figure 1. Schematic representation of the experimental protocol.

2 Figure 2. Discriminant analysis scatter plots using different number of groups and variables. NT:

3 normal training group; n-OR: intensified training group without overreaching symptoms; OR:

4 intensified training group with overreaching symptoms.

5 
1. Adlercreutz H, Harkonen M, Kuoppasalmi K, Naveri H, Huhtaniemi I, Tikkanen $\mathbf{H}$, Remes K, Dessypris A, and Karvonen J. Effect of training on plasma anabolic and catabolic steroid hormones and their response during physical exercise. International journal of sports medicine 7 Suppl 1: 27-28, 1986.

2. Armstrong LE, and VanHeest JL. The unknown mechanism of the overtraining syndrome: clues from depression and psychoneuroimmunology. Sports medicine 32: 185-209, 2002.

3. Borg G. Perceived exertion as an indicator of somatic stress. Scand J Rehabil Med 2: 92-98, 1970.

4. Bosquet $\mathbf{L}$, Leger $\mathbf{L}$, and Legros $\mathbf{P}$. Blood lactate response to overtraining in male endurance athletes. European journal of applied physiology 84: 107-114, 2001.

5. Bosquet L, Merkari S, Arvisais D, and Aubert AE. Is heart rate a convenient tool to monitor over-reaching? A systematic review of the literature. British journal of sports medicine 42: 709-714, 2008.

6. Brodde OE, Daul A, and O'Hara N. Beta-adrenoceptor changes in human lymphocytes, induced by dynamic exercise. Naunyn-Schmiedeberg's archives of pharmacology 325: 190-192, 1984.

7. Cheng B, Kuipers H, Snyder AC, Keizer HA, Jeukendrup A, and Hesselink M. A new approach for the determination of ventilatory and lactate thresholds. International journal of sports medicine 13: 518-522, 1992.

8. Chmura J, and Nazar K. Parallel changes in the onset of blood lactate accumulation (OBLA) and threshold of psychomotor performance deterioration during incremental exercise after training in athletes. International journal of psychophysiology : official journal of the International Organization of Psychophysiology 75: 287-290, 2010.

9. Costill DL, Flynn MG, Kirwan JP, Houmard JA, Mitchell JB, Thomas R, and Park SH. Effects of repeated days of intensified training on muscle glycogen and swimming performance. Medicine and science in sports and exercise 20: 249-254, 1988.

10. Coutts AJ, Slattery KM, and Wallace LK. Practical tests for monitoring performance, fatigue and recovery in triathletes. Journal of science and medicine in sport / Sports Medicine Australia 10: 372-381, 2007.

11. Duffield R, Dawson B, Pinnington HC, and Wong P. Accuracy and reliability of a Cosmed K4b2 portable gas analysis system. Journal of science and medicine in sport / Sports Medicine Australia 7: 11-22, 2004.

12. Dupuy O, Lussier M, Fraser S, Bherer L, Audiffren M, and Bosquet L. Effect of overreaching on cognitive performance and related cardiac autonomic control. Scandinavian journal of medicine \& science in sports 2012.

13. Dupuy O, Renaud M, Bherer L, and Bosquet L. Effect of functional overreaching on executive functions. International journal of sports medicine 31: 617-623, 2010.

14. Favret F, and Richalet JP. Exercise and hypoxia: the role of the autonomic nervous system. Respiratory physiology \& neurobiology 158: 280-286, 2007.

15. Fletcher $\mathbf{H}$, and Munson WA. Loudness, Its Definition, Measurement and Calculation. J Acoust Soc Am 5: 82-108, 1933.

16. Fry RW, Morton AR, Garcia-Webb P, Crawford GP, and Keast D. Biological responses to overload training in endurance sports. European journal of applied physiology and occupational physiology 64: 335-344, 1992. 

Jeukendrup AE. Time course of performance changes and fatigue markers during intensified training in trained cyclists. Journal of applied physiology 93: 947-956, 2002.

18. Halson SL, and Jeukendrup AE. Does overtraining exist? An analysis of overreaching and overtraining research. Sports medicine 34: 967-981, 2004.

19. Hedelin R, Kentta G, Wiklund U, Bjerle P, and Henriksson-Larsen K. Short-term overtraining: effects on performance, circulatory responses, and heart rate variability. Medicine and science in sports and exercise 32: 1480-1484, 2000.

20. Hedelin R, Wiklund U, Bjerle P, and Henriksson-Larsen K. Cardiac autonomic imbalance in an overtrained athlete. Medicine and science in sports and exercise 32: 15311533, 2000.

21. Hynynen E, Uusitalo A, Konttinen N, and Rusko H. Cardiac autonomic responses to standing up and cognitive task in overtrained athletes. International journal of sports medicine 29: 552-558, 2008.

22. Jeukendrup AE, Hesselink MK, Snyder AC, Kuipers H, and Keizer HA. Physiological changes in male competitive cyclists after two weeks of intensified training. International journal of sports medicine 13: 534-541, 1992.

23. Jost $\mathbf{J}$, Weiss $\mathbf{M}$, and Weicker $\mathbf{H}$. Comparison of sympatho-adrenergic regulation at rest and of the adrenoceptor system in swimmers, long-distance runners, weight lifters, wrestlers and untrained men. European journal of applied physiology and occupational physiology 58: 596-604, 1989.

24. Klecka WR. Discriminant Analysis. In: Quantitative Applications in the Social Sciences Series, edited by Thousand Oaks CSage Publications, 1980.

25. Lehmann M, Baumgartl P, Wiesenack C, Seidel A, Baumann H, Fischer S, Spori U, Gendrisch G, Kaminski R, and Keul J. Training-overtraining: influence of a defined increase in training volume vs training intensity on performance, catecholamines and some metabolic parameters in experienced middle- and long-distance runners. European journal of applied physiology and occupational physiology 64: 169-177, 1992.

26. Lehmann M, Dickhuth HH, Gendrisch G, Lazar W, Thum M, Kaminski R, Aramendi JF, Peterke E, Wieland W, and Keul J. Training-overtraining. A prospective, experimental study with experienced middle- and long-distance runners. International journal of sports medicine 12: 444-452, 1991.

27. Lehmann M, Foster C, Dickhuth HH, and Gastmann U. Autonomic imbalance hypothesis and overtraining syndrome. Medicine and science in sports and exercise 30: 11401145, 1998.

28. Lehmann M, Mann H, Gastmann U, Keul J, Vetter D, Steinacker JM, and Haussinger D. Unaccustomed high-mileage vs intensity training-related changes in performance and serum amino acid levels. International journal of sports medicine 17: 187192, 1996.

29. Mackinnon LT, Hooper SL, Jones S, Gordon RD, and Bachmann AW. Hormonal, immunological, and hematological responses to intensified training in elite swimmers. Medicine and science in sports and exercise 29: 1637-1645, 1997.

30. Meeusen R, Duclos M, Gleeson M, Rietjens G, Steinacker J, and Urhausen A. Prevention, diagnosis and treatment of the Overtraining Syndrome. Eur J Sport Sci 6: 1-14, 2006.

31. Nederhof E, Lemmink K, Zwerver J, and Mulder T. The effect of high load training on psychomotor speed. International journal of sports medicine 28: 595-601, 2007.

32. Nederhof E, Lemmink KA, Visscher C, Meeusen R, and Mulder T. Psychomotor speed: possibly a new marker for overtraining syndrome. Sports medicine 36: 817-828, 2006. 
33. Pedersen OJ, Lyregaard PE, and Poulsen TE. The round robin test on evaluation of loudness level of impulsive noise 1977.

34. Port E. Über die Lautstärke einzelner kurzer Schallimpulse. Acustica 212-223, 1963.

35. Purvis D, Gonsalves S, and Deuster PA. Physiological and psychological fatigue in extreme conditions: overtraining and elite athletes. $P M \& R$ : the journal of injury, function, and rehabilitation 2: 442-450, 2010.

36. Pyne DB, Boston T, Martin DT, and Logan A. Evaluation of the Lactate Pro blood lactate analyser. European journal of applied physiology 82: 112-116, 2000.

37. Robinson DW, and Dadson RS. A re-determination of the equal-loudness relations for pure tones. Brit J Appl Phys 7: 166-181, 1956.

38. Satz P. Brain reserve capacity on symptom onset after brain injury: a formulation and review of evidence for threshold theory Neuropsychol 7: 273-295, 1993. 39. Snyder AC, Kuipers H, Cheng B, Servais R, and Fransen E. Overtraining following intensified training with normal muscle glycogen. Medicine and science in sports and exercise 27: 1063-1070, 1995.

40. Stevens J. Applied Multivariate Statistics for the Social Sciences. Hillsdale: 1992.

41. Thomas L, and Busso T. A theoretical study of taper characteristics to optimize performance. Medicine and science in sports and exercise 37: 1615-1621, 2005.

42. Tohmeh JF, and Cryer PE. Biphasic adrenergic modulation of beta-adrenergic receptors in man. Agonist-induced early increment and late decrement in beta-adrenergic receptor number. The Journal of clinical investigation 65: 836-840, 1980.

43. Urhausen A, Gabriel HH, and Kindermann W. Impaired pituitary hormonal response to exhaustive exercise in overtrained endurance athletes. Medicine and science in sports and exercise 30: 407-414, 1998.

44. Urhausen A, Gabriel HH, Weiler B, and Kindermann W. Ergometric and psychological findings during overtraining: a long-term follow-up study in endurance athletes. International journal of sports medicine 19: 114-120, 1998.

45. Urhausen A, and Kindermann W. Diagnosis of overtraining: what tools do we have? Sports medicine 32: 95-102, 2002.

46. Uusitalo AL, Huttunen P, Hanin Y, Uusitalo AJ, and Rusko HK. Hormonal responses to endurance training and overtraining in female athletes. Clinical journal of sport medicine : official journal of the Canadian Academy of Sport Medicine 8: 178-186, 1998.

47. Zavorsky GS. Evidence and possible mechanisms of altered maximum heart rate with endurance training and tapering. Sports medicine 29: 13-26, 2000. 


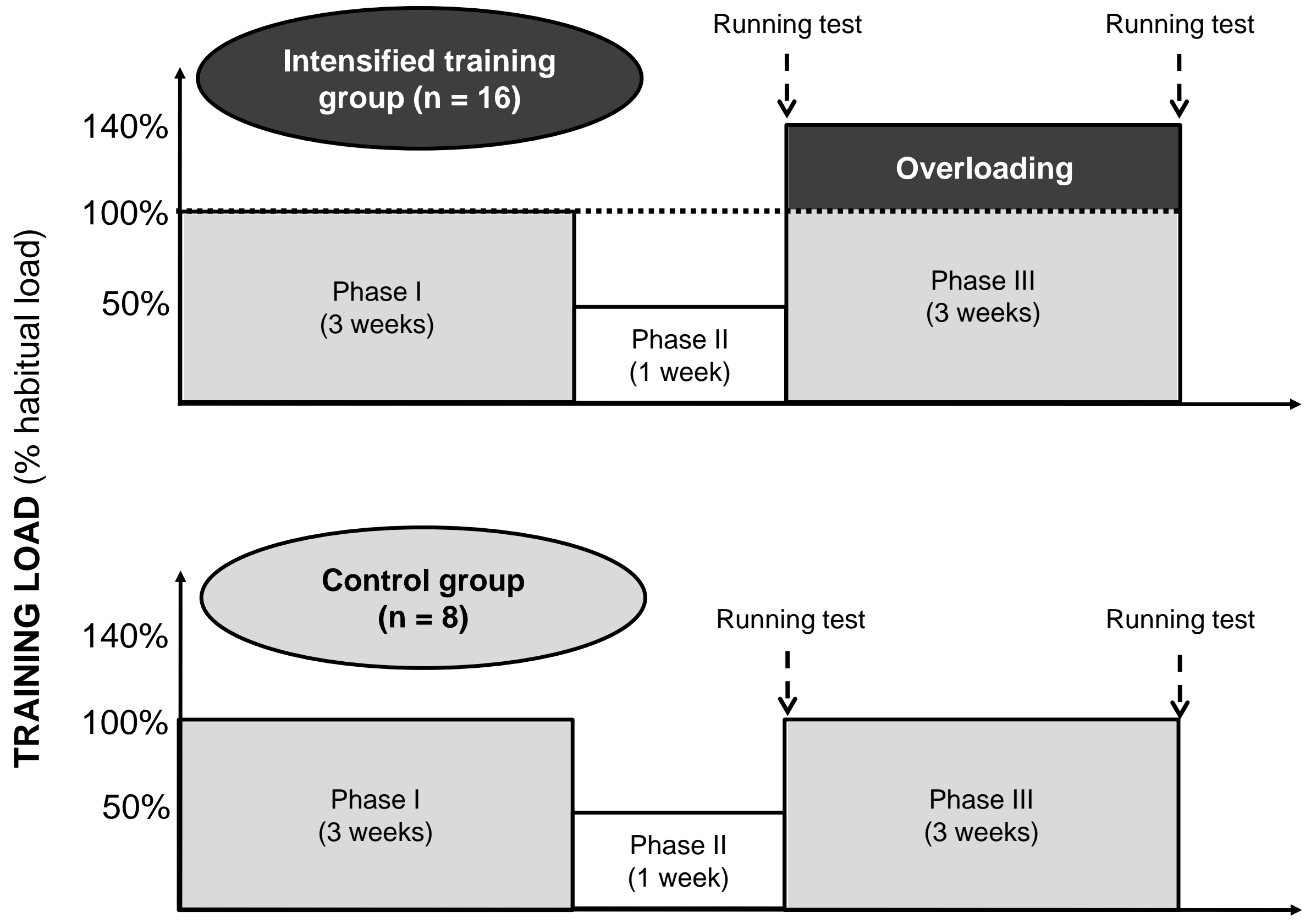


A. Discriminant analysis 1 (3 groups, 16 variables) Success rate for classification : 87.5\%

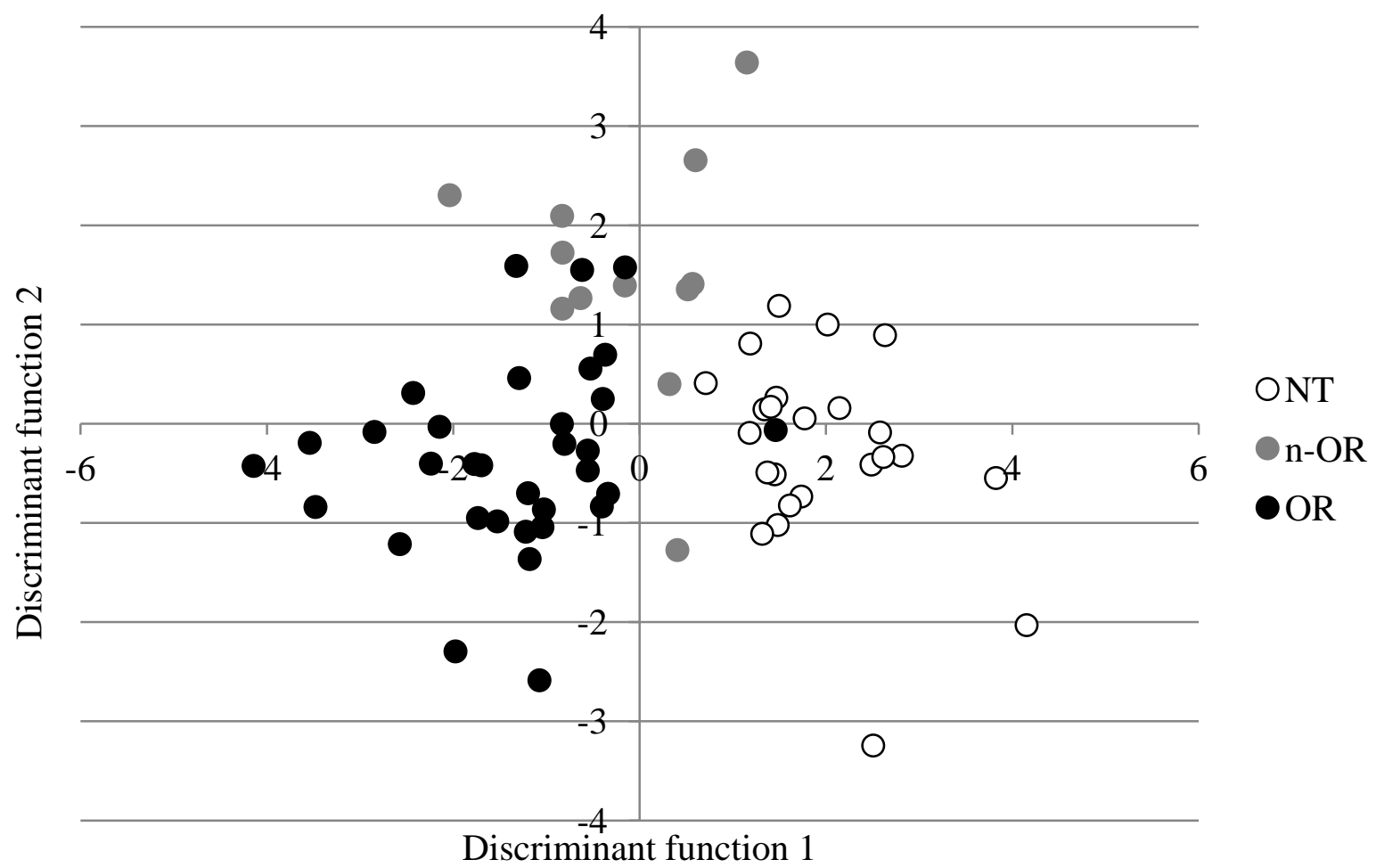

b. Discriminant analysis 2 (2 groups, 16 variables) Success rate for classification : 98.2\%

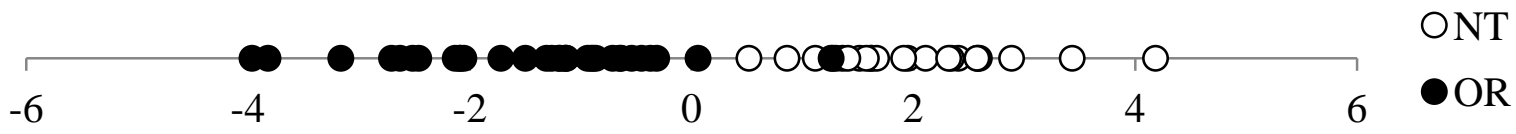

c. Discriminant analysis 3 (2 groups, 2 variables) $\quad$ Success rate for classification : 89.5\%

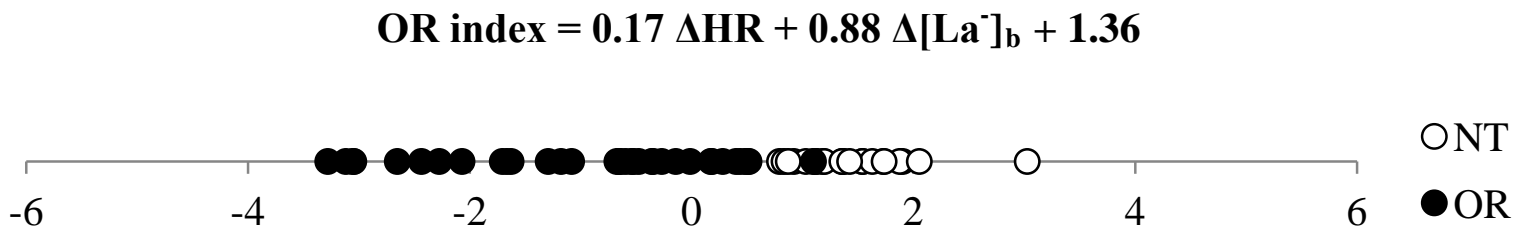




\begin{tabular}{|c|c|c|}
\hline $\begin{array}{c}\text { Subject } \\
\text { characteristics }\end{array}$ & $\begin{array}{l}\text { Normal Training group } \\
(\mathbf{n}=\mathbf{8})\end{array}$ & $\begin{array}{l}\text { Intensified Training group } \\
\qquad(\mathrm{n}=15)\end{array}$ \\
\hline Age (years) & $32.4 \pm 2.8$ & $31.0 \pm 1.4$ \\
\hline Height (cm) & $176.8 \pm 2.1$ & $178.7 \pm 1.2$ \\
\hline Weight (kg) & $69.7 \pm 2.6$ & $70.6 \pm 1.3$ \\
\hline$\dot{\mathbf{V}} \mathbf{O}_{2 \max }\left(\mathrm{ml} \cdot \mathrm{min}^{-1} \cdot \mathrm{kg}^{-1}\right)$ & $64.9 \pm 2.8$ & $62.3 \pm 1.5$ \\
\hline MAS $\left(\mathbf{k m} \cdot \mathrm{h}^{-1}\right)$ & $18.2 \pm 0.4$ & $18.3 \pm 0.2$ \\
\hline
\end{tabular}

Table 1. Selected characteristics of the two experimental groups. $\dot{\mathrm{V}}_{2 \max }$ : maximal oxygen uptake; MAS: maximal aerobic speed. Values are expressed as means \pm SEM of the means. No significant difference between both groups for all the parameters. 
a.

\begin{tabular}{|c|c|c|c|c|c|c|c|}
\hline \multirow{2}{*}{ Intensity } & \multirow{2}{*}{$\begin{array}{l}\text { Physiological } \\
\text { variables }\end{array}$} & \multicolumn{3}{|c|}{$\begin{array}{l}\text { Normal Training Group } \\
(\mathrm{NT}, \mathbf{n}=\mathbf{8})\end{array}$} & \multicolumn{3}{|c|}{$\begin{array}{c}\text { Overreached Group } \\
(\mathrm{OR}, \mathrm{n}=11)\end{array}$} \\
\hline & & Pre-Training & Post-Training & Variation & Pre-Training & Post-Training & Variation \\
\hline \multirow{4}{*}{ Low } & $\begin{array}{c}\dot{\mathrm{V}}_{\mathrm{O}_{2}} \\
\left(\mathbf{m l O}_{2} \cdot \mathbf{m i n}^{-1} \cdot \mathbf{k g}^{-1}\right)\end{array}$ & $48.8 \pm 5.0$ & $47.9 \pm 5.2$ & $-0.9 \pm 2.4$ & $49.1 \pm 1.8$ & $49.7 \pm 3.3$ & $0.6 \pm 2.1$ \\
\hline & $\left.\frac{\dot{\mathrm{V}}_{\mathrm{E}}}{(\text { L.min }}{ }^{-1}\right)$ & $92 \pm 14$ & $90 \pm 13$ & $-2 \pm 5$ & $91 \pm 11$ & $94 \pm 12$ & $3 \pm 5$ \\
\hline & $\begin{array}{c}\text { HR } \\
\text { (beats. } \min ^{-1} \text { ) }\end{array}$ & $155 \pm 11$ & $154 \pm 11$ & $-1 \pm 2$ & $152 \pm 13$ & $143 \pm 13^{* *}$ & $-8 \pm 6^{\#}$ \\
\hline & $\begin{array}{c}{\left[\mathrm{La}^{-}\right]_{\mathrm{b}}} \\
\left(\mathrm{mmol.L}^{-1}\right)\end{array}$ & $1.7 \pm 0.5$ & $1.5 \pm 0.4$ & $-0.2 \pm 0.3$ & $2.7 \pm 1.0$ & $1.9 \pm 0.8^{* *}$ & $-0.8 \pm 0.8^{\#}$ \\
\hline \multirow{4}{*}{$\mathbf{L T}$} & $\begin{array}{c}\dot{\mathrm{V}}_{\mathrm{O}_{2}} \\
\left(\mathbf{m l O}_{\mathbf{2}} \cdot \mathbf{m i n}^{-1} \cdot \mathbf{k g}^{-1}\right)\end{array}$ & $58.5 \pm 3.4$ & $58.5 \pm 1.5$ & $0.0 \pm 0.6$ & $57.4 \pm 3.9$ & $57.9 \pm 6.0$ & $0.4 \pm 3.0$ \\
\hline & $\begin{array}{c}\dot{\mathrm{V}}_{\mathrm{E}} \\
\left(\mathbf{L} \cdot \mathbf{m i n}^{-1}\right)\end{array}$ & $130 \pm 19$ & $131 \pm 14$ & $1 \pm 8$ & $126 \pm 17$ & $130 \pm 19$ & $4 \pm 5$ \\
\hline & $\begin{array}{c}\text { HR } \\
\text { (beats.min }{ }^{-1} \text { ) }\end{array}$ & $176 \pm 8$ & $175 \pm 8$ & $-1 \pm 3$ & $172 \pm 9$ & $163 \pm 9 * *$ & $-9 \pm 5^{\# \#}$ \\
\hline & $\begin{array}{c}{\left[\mathrm{La}^{-}\right]_{\mathrm{b}}} \\
\left(\mathbf{m m o l} . L^{-1}\right)\end{array}$ & $3.4 \pm 0.8$ & $3.1 \pm 0.8$ & $-0.2 \pm 0.6$ & $3.8 \pm 1.1$ & $2.5 \pm 0.7^{* *}$ & $-1.3 \pm 0.8^{\#}$ \\
\hline \multirow{4}{*}{$\begin{array}{c}\text { At } \\
\text { exhaustion }\end{array}$} & $\begin{array}{c}\dot{\mathrm{V}}_{\mathrm{O}_{2}} \\
\left(\mathbf{m l O}_{2} \cdot \mathbf{m i n}^{-1} \cdot \mathbf{k g}^{-1}\right)\end{array}$ & $61.5 \pm 3.3$ & $61.3 \pm 1.6$ & $-0.2 \pm 1.2$ & $61.0 \pm 5.2$ & $60.9 \pm 6.4$ & $-0.1 \pm 3.2$ \\
\hline & $\begin{array}{c}\dot{\mathrm{V}}_{\mathrm{E}} \\
\left(\mathbf{L} \cdot \mathrm{min}^{-1}\right)\end{array}$ & $154 \pm 17$ & $159 \pm 15$ & $5 \pm 11$ & $162 \pm 22$ & $161 \pm 23$ & $-1 \pm 11$ \\
\hline & $\begin{array}{c}\text { HR } \\
\text { (beats.min }^{-1} \text { ) }\end{array}$ & $182 \pm 13$ & $182 \pm 12$ & $0 \pm 1$ & $181 \pm 8$ & $173 \pm 8^{* * *}$ & $-8 \pm 3^{\# \# \#}$ \\
\hline & $\begin{array}{c}{\left[\mathrm{La}^{-}\right]_{\mathrm{b}}} \\
\left(\mathrm{mmol}^{-1} \mathrm{~L}^{-1}\right)\end{array}$ & $8.9 \pm 1.1$ & $9.0 \pm 0.7$ & $0.3 \pm 0.6$ & $8.1 \pm 2.0$ & $6.9 \pm 1.7^{* *}$ & $-1.2 \pm 0.2^{\# \#}$ \\
\hline
\end{tabular}


b.

\begin{tabular}{|c|c|c|c|c|c|c|c|}
\hline \multirow{2}{*}{ Intensity } & \multirow{2}{*}{$\begin{array}{c}\text { Biomechanical } \\
\text { parameters }\end{array}$} & \multicolumn{3}{|c|}{$\begin{array}{l}\text { Normal Training Group } \\
(\mathrm{n}=8)\end{array}$} & \multicolumn{3}{|c|}{$\begin{array}{l}\text { Overreached Group } \\
(\mathbf{n}=11)\end{array}$} \\
\hline & & Pre-Training & Post-Training & Variation & Pre-Training & Post-Training & Variation \\
\hline \multirow{4}{*}{ Low } & $\begin{array}{l}\text { Stride length } \\
\text { (x leg length) }\end{array}$ & $1.39 \pm 0.06$ & $1.40 \pm 0.08$ & $0.01 \pm 0.05$ & $1.36 \pm 0.05$ & $1.36 \pm 0.06$ & $0.00 \pm 0.03$ \\
\hline & $\begin{array}{c}\text { Support duration } \\
(\mathrm{ms})\end{array}$ & $243 \pm 11$ & $241 \pm 18$ & $-2 \pm 10$ & $255 \pm 17$ & $253 \pm 13$ & $-1 \pm 8$ \\
\hline & $\begin{array}{c}\text { Aerial duration } \\
\text { (ms) }\end{array}$ & $112 \pm 20$ & $116 \pm 21$ & $-1 \pm 10$ & $104 \pm 26$ & $104 \pm 22$ & $-1 \pm 10$ \\
\hline & $\begin{array}{l}\text { Maximum peak } \\
\text { vertical force } \\
\text { (x weight) }\end{array}$ & $2.63 \pm 0.24$ & $2.65 \pm 0.25$ & $0.02 \pm 0.07$ & $2.52 \pm 0.16$ & $2.50 \pm 0.15$ & $-0.02 \pm 0.07$ \\
\hline \multirow{4}{*}{$\mathbf{L T}$} & $\begin{array}{l}\text { Stride length } \\
\text { (x leg length) }\end{array}$ & $1.65 \pm 0.10$ & $1.63 \pm 0.11$ & $-0.02 \pm 0.04$ & $1.56 \pm 0.10$ & $1.54 \pm 0.11$ & $-0.02 \pm 0.05$ \\
\hline & $\begin{array}{c}\text { Support duration } \\
(\mathrm{ms})\end{array}$ & $211 \pm 12$ & $211 \pm 17$ & $-1 \pm 8$ & $229 \pm 15$ & $231 \pm 13$ & $2 \pm 7$ \\
\hline & $\begin{array}{c}\text { Aerial duration } \\
(\mathrm{ms})\end{array}$ & $130 \pm 23$ & $132 \pm 24$ & $2 \pm 6$ & $120 \pm 25$ & $108 \pm 23$ & $-11 \pm 12^{\# \#}$ \\
\hline & $\begin{array}{l}\text { Maximum peak } \\
\text { vertical force } \\
\text { (x weight) }\end{array}$ & $2.83 \pm 0.33$ & $2.83 \pm 0.34$ & $0.00 \pm 0.07$ & $2.67 \pm 0.21$ & $2.60 \pm 0.17$ & $-0.07 \pm 0.11$ \\
\hline \multirow{4}{*}{$\begin{array}{c}\text { At } \\
\text { exhaustion }\end{array}$} & $\begin{array}{l}\text { Stride length } \\
\text { (x leg length) }\end{array}$ & $1.79 \pm 0.11$ & $1.76 \pm 0.12$ & $-0.03 \pm 0.05$ & $1.68 \pm 0.14$ & $1.68 \pm 0.14$ & $-0.01 \pm 0.06$ \\
\hline & $\begin{array}{c}\text { Support duration } \\
\text { (ms) }\end{array}$ & $199 \pm 10$ & $198 \pm 16$ & $-1 \pm 10$ & $214 \pm 15$ & $208 \pm 13$ & $-7 \pm 9$ \\
\hline & $\begin{array}{c}\begin{array}{c}\text { Aerial duration } \\
(\mathrm{ms})\end{array} \\
\end{array}$ & $130 \pm 20$ & $131 \pm 19$ & $2 \pm 3$ & $120 \pm 22$ & $117 \pm 20$ & $-3 \pm 10$ \\
\hline & $\begin{array}{l}\text { Maximum peak } \\
\text { vertical force } \\
\text { (x weight) } \\
\end{array}$ & $2.83 \pm 0.29$ & $2.84 \pm 0.28$ & $0.01 \pm 0.08$ & $2.70 \pm 0.21$ & $2.66 \pm 0.18$ & $-0.04 \pm 0.12$ \\
\hline
\end{tabular}

Table 2. Mean values ( \pm SD) and deltas of variation of selected physiological (a) and biomechanical parameters (b) at baseline and after the training period for the normal training group and the overreached group. The data are presented for three running intensities determined at the end of the training program: Low (13km.h-1), Lactate Threshold (LT) and at exhaustion. Each parameter is presented for the same absolute

running speed before and after the training period. $\dot{V}_{\mathrm{O}}$ : oxygen uptake; $\dot{\mathrm{V}}_{\mathrm{E}}$ : expiratory flow; HR: heart rate: [La-]b: blood lactate concentration. Significantly different from pre-training at ${ }^{*} p<0.05 ; * * p<0.01$; ${ }^{* *} p<0.001$. Significantly different from the normal training group at ${ }^{\#} p<0.05 ;{ }^{\# \#} p<0.01 ;{ }^{\# \#} p<0.001$. 


\begin{tabular}{|c|c|c|c|c|c|}
\hline \multirow{2}{*}{ Group } & \multirow{2}{*}{$\begin{array}{c}\text { Number of } \\
\text { cases }\end{array}$} & \multicolumn{3}{|c|}{ Predicted group } & Correct \\
\cline { 3 - 5 } & 24 & 24 & 0 & 0 & $100 \%$ \\
\hline NT & 24 & 2 & 10 & 3 & $66.7 \%$ \\
\hline n-OR & 15 & 1 & 3 & 29 & $87.8 \%$ \\
\hline OR & 33 & 27 & 13 & 32 & $87.5 \%$ \\
\hline Total & 72 & \multicolumn{2}{|c}{} \\
\hline
\end{tabular}

Table 3. Classification matrix of discriminant analysis 1 using 3 groups and 21 variables (DA1). Each case represented one subject for one exercise intensity. NT: subjects of the normal training group; nOR: subjects of the overload group demonstrating no clinical symptoms of overreaching; OR: subjects of the overreached group. 
a.

\begin{tabular}{|c|c|}
\hline Variable & Standardized coefficient \\
\hline$\Delta \mathrm{HR}$ & -0.74 \\
\hline$\Delta \mathrm{dS}$ & -0.61 \\
\hline$\Delta \mathrm{dA}$ & -0.58 \\
\hline$\Delta\left[\mathrm{La}^{-}\right]_{\mathrm{b}}$ & -0.47 \\
\hline$\Delta \mathrm{Lxn}$ & -0.44 \\
\hline$\Delta \mathrm{PImn}$ & -0.38 \\
\hline$\Delta \mathrm{Lyn}$ & -0.26 \\
\hline$\Delta$ MuscRPE & 0.23 \\
\hline
\end{tabular}

b.

\begin{tabular}{|c|c|c|c|c|}
\hline \multirow{2}{*}{ Group } & \multirow{2}{*}{$\begin{array}{c}\text { Number of } \\
\text { cases }\end{array}$} & \multicolumn{2}{|c|}{ Predicted group } & \multirow{2}{*}{ Correct } \\
\cline { 3 - 4 } & 24 & 24 & 0 & \\
\hline NT & 24 & 1 & 32 & $97.0 \%$ \\
\hline OR & 33 & 25 & 32 & $98.2 \%$ \\
\hline Total & 57 & \multicolumn{2}{c}{} \\
\hline
\end{tabular}

c.

\begin{tabular}{|c|c|c|c|}
\hline Step & Variable & Wilk's lambda & Significance level \\
\hline 1 & $\Delta \mathrm{HR}$ & 0.39 & 0.0000 \\
\hline 2 & $\Delta\left[\mathrm{La}^{-}\right]_{\mathrm{b}}$ & 0.31 & 0.005 \\
\hline 3 & $\Delta \mathrm{PImn}$ & 0.29 & 0.03 \\
\hline 4 & $\Delta \mathrm{dS}$ & 0.31 & 0.004 \\
\hline 5 & $\Delta \mathrm{dA}$ & 0.30 & 0.009 \\
\hline 6 & $\Delta \mathrm{Lxn}$ & 0.30 & 0.02 \\
\hline 7 & $\Delta \mathrm{Lyn}$ & 0.28 & 0.14 \\
\hline 8 & $\Delta$ MuscRPE & 0.27 & 0.20 \\
\hline
\end{tabular}

Table 4. Detailed results for the stepwise discriminant analysis using 2 groups and 21 variables (DA2): standardized canonical discriminant function coefficients (a); classification matrix (b) and summary table (c). NT: normal training group; OR: overreached group; HR: heart rate; [La $]_{\mathrm{b}}$ : blood lactate concentration; PImn: normalised maximum peak vertical force; dS: support duration; dA: aerial duration; Lxn: normalised stride length; Lyn : normalised stride largeness; MuscRPE: muscular rate of perceived exertion. 


\begin{tabular}{|c|c|c|c|c|}
\hline \multirow{2}{*}{ Group } & \multirow{2}{*}{$\begin{array}{c}\text { Number of } \\
\text { cases }\end{array}$} & \multicolumn{2}{|c|}{ Predicted group } & \multirow{2}{*}{ Correct } \\
\cline { 3 - 4 } & 24 & 23 & 1 & $95.8 \%$ \\
\hline NT & 24 & 5 & 28 & $84.8 \%$ \\
\hline OR & 33 & 28 & 29 & $89.5 \%$ \\
\hline Total & 57 & \multicolumn{2}{|c}{} \\
\hline
\end{tabular}

Table 5. Classification matrix of discriminant analysis using two groups and two variables $(\Delta \mathrm{HR}$, $\Delta\left[\mathrm{La}^{-}\right]_{\mathrm{b}}$, DA3). Each case represented one subject for one exercise intensity. NT: normal training group; OR: overreached group. 